\title{
THE PROJECTION OF A LINE SECTION UPON THE RATIONAL PLANE CUBIC CURVE.
}

BY PROFESSOR JOSEPH EUGENE ROWE.

(Read before the American Mathematical Society, April 28, 1917.)

\section{Introduction.}

THE rational plane curve of the third order, which we shall refer to as the $R^{3}$, is of the fourth class; that is, from an arbitrary point of the plane four tangents can be drawn to the curve. But if the point is selected on the $R^{3}$ itself, the tangent at the point accounts for two of these tangents, and, therefore, from such a point only two additional tangents can be drawn to the curve. A line section yields three points of the $R^{3}$ and these, in the manner just described, determine three pairs of additional tangents. An investigation of the points of a line and the six tangents so determined shows that the relations which exist among these are interesting as well as of a fundamental character.

We shall let

$$
x_{i}=a_{i} t^{3}+3 b_{i} t^{2}+3 c_{i} t+d_{i} \quad(i=0,1,2)
$$

be the parametric equations of the points of the $R^{3}$, and it has been found convenient to use the following abbreviations:

$$
\alpha=|a b c|, \quad \beta=|a b d|, \quad \beta^{\prime}=|a c d|, \quad \alpha^{\prime}=|b c d| .
$$

Also, it may be verified that the identities

$$
a_{i} \alpha^{\prime}-b_{i} \beta^{\prime}+c_{i} \beta-d_{i} \alpha=0
$$

exist among the coefficients in (1) and the Greek letters of (2).

\section{The Choice of a Line Section.}

As the parameters 0 and $\infty$ may be assigned to any two elements in a one-dimensional space, we select the line determined by the points of the $R^{3}$ whose parameters are 0 and $\infty$. From (1) it follows that the coordinates of these points are $d_{i}$ and $a_{i}$, respectively; hence the equation of the line determined by them is $|a d x|=0$, and the parameter of the third point 
of the $R^{3}$ (found by substituting from equations (1) in $|a d x|=0$ ) collinear with $a_{i}$ and $d_{i}$ is the root of

$$
\beta t+\beta^{\prime}=0 \text {. }
$$

By substituting $t=-\beta / \beta^{\prime}$ in (1) we obtain for the coordinates of the point (4)

$$
x_{i}=-a_{i}{\beta^{\prime}}^{3}+3 b_{i} \beta^{\prime 2} \beta-3 c_{i} \beta^{\prime} \beta^{2}+d_{i} \beta^{3} \quad(i=0,1,2) .
$$

The Projections of the Three Points upon the $R^{3}$.

The projection of a point $x_{i}$ upon (1) is*

$$
\begin{aligned}
|a b x| t^{4}+2|a c x| t^{3}+(|a d x| & +3|b c x|) t^{2} \\
& +2|b d x| t+|c d x|=0 .
\end{aligned}
$$

That is, if the coordinates of a point $x_{i}$ are substituted in (6), the result is a quartic in $t$ whose roots are the parameters of the points of contact of the four tangents that can be drawn from $x_{i}$ to the $R^{3}$ of (1).

By substituting $a_{i}, d_{i}$, and the coordinates (5) in (6) for $x_{i}$ we obtain

$$
\begin{gathered}
3 \alpha t^{2}+2 \beta t+\beta^{\prime}=0, \\
\beta t^{2}+2 \beta^{\prime} t+3 \alpha^{\prime}=0, \\
\left(\beta^{2}-3 \alpha \beta^{\prime}\right) t^{2}+\left(3 \alpha^{\prime} \beta-{\beta^{\prime 2}}^{2}\right)=0,
\end{gathered}
$$

whose roots are the parameters of the points of contact of the tangents to $R^{3}$ drawn from the points whose parameters are $\infty, 0$, and $-\beta^{\prime} / \beta$, respectively.

The form of equations (7)-(9), if properly interpreted, conveys a great deal of information. Evidently the roots of $(9) \dagger$ are harmonic $\ddagger$ (apolar) to 0 and $\infty$; the roots of (8) are harmonic to $\infty$ and $-\beta^{\prime} / \beta$; and the roots of (7) are harmonic to 0 and $-\beta^{\prime} / \beta$. These results we summarize in

THEOREM I. The parameters of any two of three collinear points on the $R^{3}$ are harmonic to the parameters of the points of contact of the two additional tangents that can be drawn to the $R^{3}$ from the third point.

* J. E. Rowe, Bulletin, vol. 22, No. 2, p. 75 (November, 1915).

† Observe that the order of statement in this sentence is not without purpose.

$\ddagger$ Salmon, Higher Algebra, fourth edition, p. 179. 
Also, the determinant of equations (7)-(9) vanishes, as may be seen at once from the fact that (9) may be obtained by subtracting $\beta^{\prime}$ times (7) from $\beta$ times (8). Hence* we have

THEOREM II. The parameters of the points of contact of the three pairs of tangents that can be drawn to the $R^{3}$ from three collinear points of the $R^{3}$ are harmonic to the same quadratic, or form a set in involution.

Another result which may be derived as a corollary of Theorem I we shall state as

Theorem III. Lines on a point $P$ of an $R^{3}$ cut the $R^{3}$ in pairs of residual points whose parameters are harmonic to the parameters of the points of contact of the two additional tangents drawn to $R^{3}$ from $P$.

Although Theorem III may be regarded a corollary of Theorem I, it may be established independently. Thus: Let $P\left(d_{0}, d_{1}, d_{2}\right)$ be the point and $(\kappa x) \equiv \kappa_{0} x_{0}+\kappa_{1} x_{1}+\kappa_{2} x_{2}=0$ any line on $P$. Then $(\kappa d)=0$. The parameters of the residual points cut out of (1) by $(\kappa x)=0$ are the roots of

$$
(\kappa a) t^{2}+3(\kappa b) t+3(\kappa c)=0
$$

and (10) is apolar to (8), for

$$
3(\kappa c) \beta+3(\kappa a) \alpha^{\prime}-3(\kappa b) \beta^{\prime}=0,
$$

as may be shown from (3) and the fact that $(\kappa d)=0$.

Pennsylvania State College, March, 1917.

\section{EXAMPLES OF A REMARKABLE CLASS OF SERIES.}

BY PROFESSOR R. D. CARMICHAEL.

(Read before the American Mathematical Society, April 28, 1917.)

Two-Fold and One-Fold Expression of the Properties of Functions.

1. In the development of analysis during the past generation it has frequently happened that functions have arisen which are analytic in a sector of the complex plane and in

* Salmon, Higher Algebra, fourth edition, p. 180. 